\title{
Attosecond-Resolved Electron Dynamics in Many-Electron Atoms: Quantitative Theory and Comparison with Measurements
}

\author{
Cleanthes Anthony Nicolaides \\ Theoretical and Physical Chemistry Institute, National Hellenic Research, Foundation, \\ 48 Vasileos Constantinou Avenue, 11635 Athens, Greece; caan@eie.gr
}

Received: 13 February 2018; Accepted: 27 March 2018; Published: 30 March 2018

\begin{abstract}
A variety of processes originating from the interaction of atomic or molecular N-electron states with strong and/or hypershort radiation pulses can be understood quantitatively only by first determining with good accuracy the solutions of the many-electron time-dependent Schrödinger equation (METDSE) that describe the corresponding physics. The METDSE is solvable nonperturbatively via the state-specific expansion approach (SSEA). SSEA solutions have been used, or can be used, for quantitative explanation and numerically reliable predictions of quantities that have been measured or are measurable in modern laser-driven experiments that can track, with hypershort (attosecond) time resolution, the effects of electron rearrangements in atoms and molecules. The calculations take into account in a transparent way the interplay between the phenomena and the electronic structures of the physically significant states in discrete and multichannel continuous spectra, including multiply- and inner-hole-excited resonance states. The discussion focuses on novel topics of time-resolved many-electron physics and includes a comparison of our predictions to recent quantitative measurements of attosecond-resolved generation of the profile of the $(2 s 2 p)^{1} P^{o}$ doubly excited resonance state of helium during photoionization and of the relative time delay in photoemission of the $(2 s, 2 p)$ electrons of neon.
\end{abstract}

Keywords: time-resolved hyperfast electronic processes; time-dependent Schrödinger equation; state-specific expansion approach; attosecond science

\section{Prologue}

The paper was written in response to an invitation by the editor (M. Schultze) to contribute a brief account of theoretical work and corresponding results that my colleagues Komninos and Mercouris and I have produced since the 1990s on topics that were announced for this special issue. The announcement states that the contribution may be a review "on studies of ultrafast phenomena and tools and methods to perform them". The following discussion aims at doing just that, by commenting briefly on certain of our proposals, computational tools, and numerical results on themes concerning electron dynamics in many-electron atoms and molecules that can be studied, theoretically and experimentally, with attosecond resolution.

Solving such past and future prototypical problems involves calculating and using the many-electron, time-dependent wavefunction, $\Psi(t)$, describing the physics of interest. This is obtainable by nonperturbatively solving the many-electron time-dependent Schrödinger equation (METDSE) from first principles, in terms of the state-specific expansion approach (SSEA), which was introduced in 1993-1994 [1-3].

The label "many-electron" is meant to include atoms and molecules with more than two electrons. The initial states can have arbitrary electronic structures, where the couplings may create zero-order 
single- or multi-configurational descriptions of various types of symmetry. The SSEA and the theory of electronic structures on which it is based provide the conceptual and practical framework for quantitatively treating time-dependent many-electron problems (TDMEPs) [1-3], such as those resulting from the interaction of atoms and molecules with femtosecond and attosecond pulses and have to do with hyperfast electronic processes.

\section{Introduction}

In modern studies of the interaction of hypershort and/or strong radiation pulses for a large range of wavelengths with atoms and molecules, a desideratum of crucial importance is the possibility of extracting quantitative (i.e., not just descriptive) information and understanding about nonlinear processes for different wavelengths, (e.g., high-harmonics generation) or time-resolved phenomena of hyperfast electron dynamics, where both the discrete spectrum and the multichannel continua, including resonance (autoionizing) electronic states, are involved.

As regards theory, such information and understanding can become accessible via formal constructions and computational methods that can go beyond the level of phenomenology or one-electron models and solve a variety of TDMEPs on the time axis. This endeavor depends directly on the possibility of determining and using the time-dependent solution, $\Psi(t)$, of the METDSE. The matter-field interaction contains the pulse characteristics of duration, shape, intensity, and frequency.

If the many-electron $\Psi(t)$ for a particular problem can be calculated accurately, its use within an appropriate theoretical construction allows the reliable description of the physics of interest, including, of course, quantitative predictions, the hallmark of any generally applicable theory. The same physics is obtainable via the appropriate measurement.

For ordinary ground states of neutral atoms and molecules interacting with pulsed radiation, if the field is strong, say $10^{12}$ to $10^{13} \mathrm{~W} / \mathrm{cm}^{2}$ and above, and the pulse is hypershort, say in the range of attoseconds (as) to a few decades of femtoseconds $(f s)$ (normally not too many cycles of the field), $\Psi(t)$ must, in general, be obtained nonperturbatively, taking into account, correctly and systematically, the significance of each problem's contributions to the states of both the discrete and the continuous spectra.

This criterion is satisfied by the SSEA, which is conceptually simple, yet general and transparent with respect to the interplay between dynamics and electronic structures and spectra. A brief description of this computational tool in the theoretical study of time-resolved electron dynamics is given in Section 3. For details, the reader is referred to [1-3] and their references.

Implementation of the SSEA has allowed different types of TDMEPs to be solved, with quantitative results that can be compared with a variety of experiments. For example, see [4,5] for results from the calculation and quantitative understanding of observables in the multiphoton ionization of helium by UV and EUV femtosecond pulses.

Lest it is thought from the previous statement that the SSEA is limited to two-electron systems, I note that the applications include many-electron atoms and topics of multiphoton dissociation and association of diatomics, with initial-state wavefunctions being of the closed-shell as well as the open-shell type [1-3]. For example, the SSEA calculation of $\Psi(t)$ (including state-specific correlations), allowed the quantitative prediction of the time-resolved creation during photoionization of the profiles of inner-hole autoionizing states of aluminum decaying into a multichannel continuum [6].

The significance of going beyond two-electron systems when attempting to solve the METDSE while taking into account the state-specific self-consistent fields and electron correlations for any type of electronic structures was underlined in [6] as follows:

This is clearly a desideratum for experiment as well, if progress is to be characterized by new information on a variety of real systems. For example, only a small part of what one learns about control or about the effects of electron correlation from the experimental and/or theoretical studies of the interaction of the He $1 s^{2}{ }^{1} \mathrm{~S}$ ground state with a strong electromagnetic pulse, can be transferred 
to other systems. In other words, just as in the case of the decades-old time-independent many-electron problem and the chasm between the many body problem for He and that for other larger atoms, especially in excited states, even a good calculation on the system "laser pulse-He $1 s^{2}{ }^{1} S^{\prime}$ " cannot help very much in understanding quantitatively field-induced phenomena exhibited by arbitrary ground or excited states of other atoms across the Periodic Table. ([6], pp. 1-2).

\section{Contents}

This review is divided into two parts, both of which serve as commentary.

In the first part, Section 3, I explain briefly the key features of the SSEA for solving the METDSE from a many-electron point of view [1-3].

The second part, Sections 4-6, points to experimental findings and corresponding discussions concerning prototypical ultrafast electronic processes and phenomena in N-electron atoms [7-11] and compares them to results and discussions by the Athens team published from 1996 to 2011 [1-4,11-17]. Papers [7-17] have to do with the quantitative understanding and the possibility of credible prediction of the time-dependent many-electron physics of attosecond-resolved electron rearrangements in many-electron systems.

\section{Solving the METDSE Nonperturbatively via the State-Specific Expansion Approach}

\subsection{Introduction}

The fundamental equation that contains information about the properties and phenomena that are observable when a pulse of radiation interacts with an atomic or molecular state is the METDSE:

$$
\begin{aligned}
& \boldsymbol{H}(t) \Psi(t)=i \hbar \frac{\partial \Psi(t)}{\partial t} \\
& \boldsymbol{H}(t)=\mathbf{H}+\boldsymbol{V}(\omega, t)
\end{aligned}
$$

$\mathbf{H}$ is the $\mathrm{N}$-electron field-free Hamiltonian, consisting of one- and two-electron nonrelativistic or relativistic operators. $V(\omega, t)$ is the interaction one-electron operator. Apart from time and the photon frequency $\omega$, it contains information about the polarization and intensity of the field, and about the temporal characteristics of the pulse.

The possibility of solving the METDSE for TDMEPs in real systems lies at the heart of many-electron quantum mechanics. For many decades this goal was ignored, not only because of the complexity of the TDMEP, but also because the understanding of properties and phenomena did not require quantitative knowledge of the many-electron $\Psi(t)$ of Equation (1). Instead, the focus was on the possibility of obtaining accurate solutions of the many-electron time-independent Schrödinger equation (METISE) for the calculation of properties of stationary states, including time-independent rates of transition, an activity that remains dominant, especially for $\mathrm{N}$-electron ground states.

Given the countless number of possible many-electron states and spectra and the variety of possible radiation pulses, a generally applicable theory for the ab initio solution of Equation (1) must be able to incorporate in a tractable way the important requirements of advanced theory of electronic structures, of electron correlations, and of the scattering continuum.

It should be recalled that most publications and books related to this subject ignore the serious and challenging issue of properly solving the TDMEP. In other words, no N-electron wave functions are calculated and used. Instead, these publications are exhausted at the level of either pure formalism or of phenomenology. In such categories are many discussions based on two- or three- or four-level models, where the N-electron matrix elements and energies are taken to be parameters.

In other types of investigations, the complexity of the many-electron problem is bypassed by conveniently replacing the true electronic Hamiltonian with a solvable independent electron model (IEM). IEM-type methods of solving Equation (1) are computationally convenient when applied to closed-shell (single determinantal) electronic structures. (When it comes to open-shell initial states, in most cases electron couplings are such that the zero-order description in terms of a single determinant 
is inadequate.) They may yield gross information for certain observables, analogous to the IEM that is used for the approximate solution of the METISE for closed-shell ground states. However, they cannot handle questions regarding the effects of electron correlations, of double (or multiple) excitations, and of interchannel couplings in the discrete and the continuous spectrum. Hence, unless one has a good understanding from experience or formal analysis of the situations for which the IEM may be reasonably accurate and meaningful, predictions for arbitrary systems based on IEM-type approaches are either practically impossible or unreliable.

\subsection{The State-Specific Expansion Approach (SSEA)}

For one-electron systems, the solution of Equation (1) for various problems has been achieved numerically, either via direct integration on a space-time grid or via a method of expansion over some type of one-electron basis functions. The grid method has also proven practical in certain cases involving closed-shell N-electron states, where, following the proposal and results of Kulander in the late 1980s [18,19], the formalism is simplified by assuming that the field-driven integration involves only one active electron at a time (single active electron (SAE) model). Space-time numerical integration over a grid in all dimensions of the partial differential Equation (1) has also been implemented in the case of the two-electron closed-shell ${ }^{1} S$ state. (e.g., see [20], where differences between the SAE and the full two-electron results are attributed to electron correlations).

The full grid approach is practically inapplicable to arbitrary N-electron electronic structures, i.e., beyond $\mathrm{He}$ or $\mathrm{H}_{2}$, even with the help of supercomputers. This limitation holds not only for the METDSE, but also for the eigenvalue problem of the METISE, as was recognized in the 1960s.

Evidently, the theory for solving the METDSE for arbitrary electronic structures must follow a different path. This fact determined the research program we initiated in the early 1990s. We concluded that a robust and general approach would be one that invokes the fundamental expansion principle in terms of stationary states with complex time-dependent coefficients, and capitalizes on knowledge of, and experience with, calculating the stationary state-specific $\mathrm{N}$-electron wave functions of the discrete and of the continuous spectrum that solve the METISE [1-3,21,22]:

$$
\begin{aligned}
& \mathbf{H \Psi}_{k}(q)=E_{k} \Psi_{k}(q) \\
& \mathbf{H}=\sum_{i=1}^{N} \boldsymbol{h}_{i}+\sum_{i>j}^{N} \boldsymbol{g}_{i j}
\end{aligned}
$$

$k$ labels stationary states of either the discrete or the continuous spectrum. The latter are energy-normalized. $q$ stands for the coordinates of the $N$ electrons.

In our SSEA work on the solution of Equation (1), the electron-field coupling operator in $V(\omega, t)$ has been used either in the electric dipole approximation or as the full electric operator of the multipolar Hamiltonian [23] constrained by dipole selection rules ([24] and its references).

The critical question is how to nonperturbatively solve the METDSE using $\boldsymbol{H}(t)$ and the many-electron $\mathbf{H}$ of Equations (1) and (2). For this purpose, we write the formal solution of Equation (1) as follows (here, for simplicity, I use only one-channel for the continuum):

$$
\left|\Psi(t)>=\sum_{m} a_{m}(t)\right| m>+\int_{0} b_{\varepsilon}(t) \mid \varepsilon>d \varepsilon
$$

where $\mid m>$ are the stationary states of the discrete spectrum and $\mid \varepsilon>$ are those of the continuous spectrum, including possible resonances. The scattering wave functions are energy-normalized according to $\left\langle\varepsilon \mid \varepsilon^{\prime}\right\rangle=\delta\left(\varepsilon-\varepsilon^{\prime}\right)$. The complex coefficients represent the projection of the wavepacket $\mid \Psi(t)>$ onto the corresponding stationary states.

The objective of the SSEA is to represent the $\mid m>$ and $\mid \varepsilon>$ of Equation (3) by the state-specific wavefunctions that are relevant to the problem of interest, and to correctly compute the time-dependent coefficients. The latter task is accomplished by solving the coupled equations that result from 
substituting Equation (3) into the METDSE. The calculation is transparent for each trial set of state-specific wavefunctions, and is carried out by adjusting parameters such as the time step or the range of the free-electron energy and the number of points (solutions) in it, so as to achieve good convergence. This endeavor requires calculating energies and bound-bound, bound-free, and free-free $\mathrm{N}$-electron matrix elements $[1,2,21,22,24]$.

One of the advantages of constructing expansion (3) in terms of wavefunctions that are calculated separately for each state, in terms of their own zero-order and correlation function spaces, is that in the process of solving, one can monitor and evaluate with transparency and economy the dependence of the evolution of $\Psi(t)$ on each $\mid m>$ and $\mid \varepsilon>$. In other words, unlike other methods that are often used for the approximate solution of Equation (1), the SSEA is not a "one-shot" algorithm that is applied in the same way to different cases. Instead, the methodology involves systematic expansion in terms of state-specific N-electron wavefunctions for the discrete and the continuous spectrum, according to the physics of each problem and the observed convergence of the calculation.

Furthermore, the SSEA is not limited to special electronic structures that are described by a single determinant, nor does it ignore the variety of effects of electron correlations, multiply excited resonances, and interchannel couplings.

Once the expansion coefficients of Equation (3) have been obtained from the solution of Equation (1), it is straightforward to use them, together with the stationary wavefunctions, in order to unambiguously compute the dynamics of interest as a function of time during and at the end of the pulsed interaction. This simple and direct way of quantitatively recognizing the correspondence of the stationary states with the field-induced dynamics is a hallmark of the SSEA. Its utility has been demonstrated in a variety of applications to difficult and computationally demanding TDMEPs, where, among other things, insight and experimentally relevant quantitative information has been gained by using the SSEA coefficients, as, for example, in cases of photoelectron angular distributions, above threshold ionization, the contribution of Rydberg states in multiphoton ionization, and the relative time delay in the emission of photoelectrons from different subshells (see [1-6] and Section 6 of this paper). The solution of the METDSE according to the SSEA takes into account the following:

* The zero-order features of electronic structures of initial, intermediate, and final states. These can be calculated at the Hartree-Fock (HF) or multiconfigurational HF (MCHF) level (see Equation (4) below).

* The dominant electron correlations for those states (initial, intermediate, or final) where this is necessary.

* The contribution of perturbed or unperturbed Rydberg levels, and of doubly or even multiply and inner-hole excited states.

* The contribution of the continuous spectrum of energy-normalized, channel-dependent scattering states, without or with resonance states.

* The interchannel coupling.

It is important to add that the structure of the SSEA allows the use of relativistic wavefunctions as well.

Molecules

As regards molecules, the SSEA has been implemented only for the purpose of treating special themes involving vibrational states and dissociation continua in diatomics, where the smooth continuum has been described in terms of either box-normalized basis sets $[1,25]$ or numerical energy-normalized scattering states [26]. It has not yet been applied to problems of time-resolved electron dynamics in molecules, for which descriptive discussions can be found (e.g., in [27]). Given our limited human resources, our prospects of tackling such problems in molecules at the level of rigor that has been demonstrated for atoms via the SSEA are meager. However, the following comment is relevant. 
Tracking the hyperfast motions of electrons in molecules while discerning their effects from those caused by the other degrees of freedom is not easily amenable to rigorous computational analysis by accurately solving the METDSE, especially when the molecule is excited by a pulse of relatively short wavelength to a multitude of interacting states and channels. Of course, approximate treatments can, in principle, produce rough answers. As regards possible SSEA-type approaches, where the inclusion of excited states must be done carefully and economically in terms of separately optimized function spaces, the area is essentially open. Here, one fundamental difficulty lies in the proper and reliable calculation of electronic scattering wavefunctions. For diatomics, incorporating such wavefunctions into the SSEA expansion can, in principle, be done as a symmetry-adapted superposition of orbital configurations containing the scattering orbital obtained in the potential of the (N-1)-electron ion. Work and references regarding the calculation of the scattering orbital can be found, e.g., in [28]. However, significant progress is still needed toward the goal of treating polyatomics, with respect to both the electronic excited discrete and resonance states and the accurate representation and use of multichannel continua for different geometries of potential energy surfaces.

\subsection{The Meaning of the Term "Electron Correlations"}

As the title of our paper [14] shows, the concept of electron correlation was at the core of our initial considerations and proposals aiming to connect the prospects of observable effects of electron dynamics to spectroscopy with attosecond resolution. At that time (2001), published statements regarding the possible connection of attosecond pulses to electron dynamics had not yet identified specific concepts and effects whose interrogation would require attosecond resolution. The general argument regarding the relevance of attosecond pulses simply invoked the classical picture of a $1 \mathrm{~s}$ Bohr electron of hydrogen going around a circle with a period of about 150 as.

In order to explore the prospects of practical spectroscopic use of attosecond pulses for issues of time-resolved effects of electron dynamics on spectra, we identified as suitable candidates the doubly excited states (DESs) in the continuous spectrum of helium $[1-3,14,15]$. Helium is attractive to both theoreticians and experimentalists for practical reasons. By now, there are a number of publications in the area of attophysics with results on time-resolved spectra involving DESs of helium.

In recent years, in the growing literature on themes of the nascent field of attosecond physics [29], the term electron correlation is often used to describe a feature that is inextricably linked to electron dynamics studied with high temporal resolution. In order to avoid misunderstanding as to the meaning of this term, which is used in our work, I comment as follows:

The term is not the same as "electron interactions", whose meaning is related to the obvious fact that there are two-electron operators of the atomic (molecular) Hamiltonian, nonrelativistic or relativistic. In other words, if, say, a calculation is carried out in the absence of the Coulomb operator $1 / r_{12}$, the result represents an artificial situation. In our theoretical and computational work on the electronic structure and properties of atoms and molecules, electron correlation is defined and computed in terms of wave functions, as outlined below.

The term electron correlation was coined by Wigner in his theory of the electron gas for metals in 1934 [30]. It was later picked up and first used in the literature of quantum chemistry in 1952 by Taylor and Parr [31], in their configuration-interaction study of the $1 s^{2}{ }^{1} S$ state of helium. They defined it as the part of the exact solution of Equation (2) that remains after calculating the single-configuration HF wave function and energy. The same definition was later emphasized by Löwdin [32] in his review article on electron correlation, which dealt with solving the eigenvalue of Equation (2) for ground states.

The above definition is translated to the following standard equations of quantum chemistry:

$$
\Psi=\Phi^{H F}+X^{c o r r}, E=E^{H F}+E^{\text {corr }}
$$


where $\Psi$ and $E$ satisfy Equation (2). Equations (4) are valid for those states for which the HF equations are solvable. Furthermore, they are useful for those states for which the HF wave function is overwhelmingly dominant in the zero-order description of the exact eigenfunction. This normally occurs in many ground states of atoms and many closed-shell molecules in equilibrium. Therefore, in all those cases, and especially for ground states, the MEP becomes equivalent to the electron correlation problem defined by Equation (4).

When it comes to complicated cases of electronic structures with open subshells or of excited states, including resonance (autoionizing) states, where the mixing coefficients of a couple of configurations are large, the description of electron correlation in terms of Equation (4) has to be modified. Now, it is more meaningful and much more economical to compute the zero-order wave function as a self-consistent multiconfigurational expansion based on the state-specific "Fermi-sea", and to proceed with calculating the most important remaining electron correlation terms for the property of interest, according to the state- and property-specific (SPS) theory [21,22].

The concept of the "Fermi-sea" was introduced in the early 1970s in order to describe, in zero-order, classes of electronic structures with heavy configurational mixings, and to allow the development of an efficient theory of electron correlation for ground and excited states, and of field-induced properties and processes. Its nature, its background, and its utility are discussed in [21,22].

Accordingly, the form in which the state-specific stationary wave functions, $\Psi_{n}$, are defined, computed, and used, is [21,22]:

$$
\begin{aligned}
& \Psi_{n}=a_{0} \Psi_{n}^{0}+\sum_{i=1}^{K} a_{i} X_{n}^{i}, \quad K \rightarrow \infty \\
& \equiv a_{0} \Psi_{n}^{0}+X_{n}^{\text {corr }} \\
& a_{0}^{2}+\sum_{i=1}^{K} a_{i}^{2}=1, \quad K \rightarrow \infty
\end{aligned}
$$

$\Psi_{n}^{0}$ is, in general, a self-consistently optimized multiconfigurational wavefunction containing the Fermi-sea configurations, and $X_{n}^{i}$ are the remaining symmetry-adapted correlation configurations containing either one, two, or three, etc., virtual excitations. In practice, the virtual (correlation) orbitals are represented by analytic orbitals that are optimized variationally.

The SPS criterion for an economic calculation is that $a_{0}$ must be very close to unity. The number and nature of the correlation terms in the sum of Equation (5) then depend on the property of interest and on the level of accuracy required for meaningful comparison with the experiment in [21,22].

Here I should add that the wavefunctions of electronic excited states are often highly mixed due mainly to valence-Rydberg-continuum state interactions. Therefore, their treatment requires a special advanced theory of perturbed spectra and channel mixings, which is applicable (and has been applied) to arbitrary electronic structures $[1,2,21,22,33,34]$.

The main argument of the SPS theory $[21,22]$ is that in order to accurately compute the matrix element of an operator that represents a particular property other than the total energy, what matters most is the "optimal matching" of the necessarily approximate wavefunction with the characteristics of the operator, and not whether the total energy has been obtained to a very good approximation.

Of course, for some operators the degree of convergence of the two calculations (first the total energy and then another property) is normally in harmony, especially for ground states. In general, however, the aforementioned optimal matching is not guaranteed by the criterion of the accuracy of the total energy, since non-negligible contributions to the value of the property may come from components of the wavefunction that do not contribute significantly to the total energy. These are configurations that have small coefficients, whilst their orbital radials are evidently not optimized with respect to the matrix element of interest. Yet, they are responsible for cumulative effects involving constructive or destructive contributions, with quantitative consequences. 
The preceding observations suggest that, in general, when it comes to calculating properties and phenomena, the treatment of the omnipresent MEP need not rely exclusively on huge calculations that first obtain a very accurate total energy, especially since these are most often impractical for problems involving field-induced nonstationary states. Instead, in addition to the basics of the energy criterion, it is rewarding to also focus on and develop an understanding of the characteristics of the $\mathrm{N}$-electron wavefunction(s) with respect to the state-specific self-consistent fields and the corresponding state-specific electron correlations $[3,21,22]$. For this purpose, it is essential for the theory not only to be formally correct and general, applicable to states of all kinds, but also to be implementable in terms of adequately optimized and numerically accurate function spaces [3,21,22].

\subsection{Other Computational Schemes for Solving the METDSE for Problems of Atomic (Molecular) States Interacting with Ultrashort and/or Strong Pulses}

As was already stated in the previous sections, the aim of the present paper is to present elements of the theory and methods of the SSEA, and of quantitative results that the Athens team has produced in connection with problems that fall in the domain of attophysics. Nevertheless, it may be of interest to the reader to cite other methods that have been proposed and used to solve the METDSE. Much of the text that follows is taken from [3].

As new experimental possibilities for the study of phenomena resulting from the interaction of atomic and molecular states with short and/or strong pulses were emerging, the introduction in 1994 of the SSEA [1,2] was linked to the need at that time to develop theory and many-electron methods of computation that are suitable for nonperturbatively solving the METDSE, beyond the cases of one- and two-electron ground states interacting with ultrashort and/or strong pulses. Regardless of the type of physical problem, in pursuing such a goal, the structure of the formalism must allow, in a practical way, the calculation of effects due to the state-specific features of the pertinent electronic structures and electron correlations. This requirement is analogous to, but much more complex and demanding than, that of many-electron problems of quantum chemistry on the energy axis.

Subsequent publications by a number of other groups have also presented methods aiming to provide solutions of the METDSE for many-electron systems. A recent review of a number of such methods was given by Ishikawa and Sato [35]. Its subsections are titled "Direct solution of the TDSE", "Multiconfiguration self-consistent-field (MCSCF) approach", "Time-dependent configuration interaction (TDCI) method", "Time-dependent multiconfiguration Hartree-Fock (TDMCHF) method", "Time-dependent complete active-space self-consistent field (TD-CASSCF) method", "Time-dependent coupled-cluster method", and "R-matrix approach". In a note added in proof, the authors stated that their review did not cover the SSEA.

I comment briefly on a few of these methods, starting with the time-dependent R-matrix (TDRM) theory [36-39]. The TDRM was first outlined by Burke and Burke in 1997 [36]. The fact that it was then applied only to a toy model, multiphoton ionization of a one-dimensional model, is testimony to the difficulties and high demands that such calculations have when it comes to problems involving real polyelectronic atoms or molecules. In a recent publication [39], the TDRM method was applied to a novel problem that is discussed in Section 6, namely the determination of the relative time delay in the $(2 s, 2 p)$ photoionization of neon.

Given the experience with advanced electron correlation methods for the ground and low-lying discrete stationary states and the convenience of available computer programs of computational quantum chemistry that are applicable to solving the METISE, in recent years many research groups have entered the area of solving the METDSE for certain problems by converting known time-independent methods into time-dependent ones in terms of expansions with time-dependent coefficients. For example, one is the TDMCHF (e.g., [40-42]), and another is the TDCI (e.g., [43,44]).

Even when the initial-state electronic structure is favorable for their formal implementation (i.e., single-determinantal closed-shell ground states), methods such as those in [40-45] express the content of the SSEA only in an approximate way. However, as I already mentioned in Section 3.2, 
for the time being approximate treatments may indeed be necessary for the study of molecules. They are constrained by the necessary truncation of the basis function space and by the corresponding uncertainties and inaccuracies in descriptions of the excited states in the discrete and, especially, the continuous spectrum, including Rydberg series (unperturbed or perturbed) and multiply excited resonances. In other words, when it comes to excited states, CI methods using a common basis set produce excited roots that need not constitute a good representation of excited electronic states of the discrete spectrum, or of the multichannel continuum, especially if the function space of virtual excitations is truncated significantly.

For example, consider the "TDCI singles" method described in [44]. It is formulated as an expansion over a set of virtual excitations from a zero-order Hartree-Fock determinant, which includes only one-electron excitations. Yet, even for simple closed-shell initial states (let alone open-shell, heavily mixed wavefunctions), ignoring the presence of doubly excited states, either in the physically significant portion of the spectrum or in the contribution of the character of the excited-state wavefunctions, introduces into the method and into the corresponding results a drastic and uncontrollable uncertainty. Even in the simple case of one-photon transition amplitudes between discrete states, it has been known since the early 1970s that, although the matter-field interaction is represented by a one-electron operator, orbital relaxation (upon excitation) and electron correlations imply that both singly and doubly (at least) excited configurations must be included in the CI wavefunctions; see the discussion on the first-order theory of oscillator strengths (FOTOS) in [21,22]. In fact, this is mandatory when valence-Rydberg-scattering state mixing is important in a portion of the spectrum that may contribute to the time-dependent dynamics.

In general, for strong fields and nonperturbative situations, the degree of reliability of CI-type approximations to the SSEA will be limited, regardless of the availability of hugely increased computer power, unless the contribution of the multichannel continuous spectrum is taken into account consistently and in a way that is mathematically justifiable. Here, one must remember that when a molecule is subjected to strong fields, channels of dissociation may compete with those of ionization.

Finally, I refer to coupled-channel methods such as those published in [46,47]. As in the SSEA, where the coupled equations can reach huge numbers due to the energy-discretization of the multichannel continuum in terms of energy-normalized scattering states (e.g., [6]), coupled-channel methods such as those of [46,47] also have to deal with such issues. In this context, I note that the recent work of Majety, Zielinski, and Scrinzi [47] deals with the four-electron beryllium, for which they stated that a multichannel description is needed. It is worth pointing out that the four-electron, multiconfigurational, multichannel problem was first solved about 25 years ago in terms of the SSEA [1]. It had to do with the interaction of laser pulses with the ground state of $\mathrm{Li}^{-}$, i.e., with a state of a negative ion where near-degeneracy effects, i.e., $2 s^{2}-2 p^{2}$, must be included in zero-order, as in the case of $B e$. The demonstration of the theory involved the continuous spectrum with two open channels, whose thresholds are the $L i 1 s^{2} 2 s^{2} S$ and $1 s^{2} 2 p^{2} P^{o}$ states.

\section{Time-Resolved Many-Electron Physics of Hyperfast Electron Rearrangements}

In recent publications by Kaldun and Blättermann et al. [7,8], by Krausz [9], and by Isinger et al. [10], there is discussion and presentation of results of new experiments on time-resolved hyperfast electron dynamics in many-electron systems, made possible through the application of modern techniques of spectroscopy with attosecond resolution. These measurements and the corresponding implications are directly related to, and in harmony with, our proposals and theoretical results that were produced in the ' 00 s, based on time-dependent formalisms that allow the practical use of many-electron wavefunctions [1-6,11-17]. In the following paragraphs, I recall how these compare with the results and discussions in [7-11]. 


\section{Time-Resolved Coherent Excitation and Decay of Strongly Correlated Autoionizing States}

Over many decades, information on properties of resonance (autoionizing) states inside the continuous electronic spectra of real N-electron atoms and molecules has been obtained, to various degrees of accuracy, on the energy axis (e.g., energy positions, partial and total energy widths, photoionization cross-section in the energy region where a resonance is present, perturbation by an external field). This area of research has seen significant experimental and theoretical progress. Regarding theory, the fundamentals of resonance-state theory are settled. However, when it comes to practical implementation for problems involving complex excited electronic structures and multichannel continua, what is needed in addition to the fundamentals is the possibility of reliable calculations. Indeed, this direction contains many possibilities for application to complex situations.

In the mid-1990s, a new direction in the investigation of N-electron autoionizing (resonance) states was initiated. It was based on the many-electron calculation of the N-electron $\Psi(t)$ via the SSEA on the time axis $[12,13]$, a possibility that provides a different type of insight into their properties.

For the treatment of autoionization, the Hamiltonian is field-free and time-independent, $\mathbf{H}$. The initial state is an N-electron wavepacket, $\Psi_{0}$, which is square-integrable at $t=0$ and has energy $E_{0}=\left\langle\Psi_{0}|\mathbf{H}| \Psi_{0}\right\rangle$. The main goal in [12,13] was to calculate the time-resolved survival probability against electron emission, $\left|<\Psi_{0}\right| \Psi(t)>\left.\right|^{2}$, from which information such as the lifetime, or the energy width corresponding to exponential decay of the decaying state, can also be obtained. The temporal resolution with which $\left|<\Psi_{0}\right| \Psi(t)>\left.\right|^{2}$ is obtained can be refined down to the attosecond scale.

The prototypical cases that were studied fall into two categories of processes of dissipation into the electronic continuum:

(1) One-electron tunneling from a core-excited shape resonance, $H e^{-} 1 s 2 p^{24} P$, according to $\left.H e^{-} 1 s 2 p^{24} P^{\text {auto detachment }} \rightarrow \mathrm{He} 1 s 2 p^{3} P^{o}+\varepsilon p\right]^{4} P[12]$.

This decay process is due mainly to the state-specific self-consistent field changing from initial to final state, and can be (was) calculated as such.

(2) Two-electron rearrangement. This is the "mechanism" that normally dominates nonrelativistic or relativistic autoionization. For example, an ab initio calculation produced the time-resolved decay curve and the lifetime, $3.5 \times 10^{-14} \mathrm{~s}$, of the doubly excited state Ca KL3 $p^{6} 3 d 5 p^{3} F^{o}$ [13].

The results provided, for the first time, quantitative results for real N-electron systems regarding the time-resolved regimes of both the exponential decay and, most importantly, the nonexponential decay (NED) [12,13]. I note that the states were not chosen haphazardly. Instead, their choice was based on the theoretical results and proposal published in 1977 [48], according to which, for NED of an isolated decaying state to acquire a magnitude of possible physical significance, the energy, E, of the state must be very close to threshold and the ratio $\mathrm{R}=\mathrm{E} / \Gamma$ must be close to unity. ( $\Gamma$ is the width of the isolated narrow resonance state.) For a recent application of the results of [48] to radioactivity and carbon dating, see [49].

The insight and computational experience gained from the ab initio calculation of the time-dependent wave functions of autoionizing states, including the effects of electron correlation and of their time-resolved decay [12,13], served as input for the theoretical and computational work we initiated immediately after the important publications by Hentschel et al. [50] and Paul et al. [51] concerning the generation of attosecond pulses and trains of attosecond pulses.

Specifically, in 2002, we published the proposal-which was supported by solutions of the METDSE from first principles-that the then emerging prospects of new electronic spectroscopic techniques that can interrogate electron dynamics on the attosecond time scale could find fertile ground in the study of strongly correlated excited states in the continuum and the ensuing electron rearrangements $[14,15]$. It was pointed out that, whereas applying femtosecond spectroscopy provides information about nuclear motion, where the "motion" is described classically in terms of positions on potential energy surfaces, in the case of electrons in atoms or molecules, the time-resolution of electronic motion on the attosecond scale can be described in terms of properties of $\mathrm{N}$-electron wave functions and their superpositions. 
The understanding and treatment of such dynamics engages the following concepts and computable quantities:

* The main symmetry-adapted orbital configurations obtained in state-specific self-consistent fields, the corresponding physically relevant transition amplitudes, and the "geometries" of the electron distributions in correlating configurations, and

* The dominant state-specific electron correlations as determined by the degree of configurational mixing $[3,14]$.

As we wrote in [15], "The problem was formulated around the following two questions, the first one being more general.

"(1) Is it possible to probe the electronic structure in such a way as to extract from it meaningful information on dynamics that can be resolved within the attosecond regime? If so, what is an observable process that can reflect the concept of 'motion' of electrons?

(2) Considering the first question, given the quantum mechanical formalism of stationary states and of corresponding eigenfunctions of $3 \mathrm{~N}$ coordinates, how are we to probe and reveal the possible connection of electronic structure to atomic dimensions, to hypershort time scales and to electron correlation?" ([15], p. 2).

The case for which the first calculations were carried out was the coherent preparation and decay of the strongly correlated low-lying ${ }^{1} P^{o}$ DESs of helium. Their theoretical preparation was effected by letting two femtosecond pulses interact either with the ground state, $1 s^{2}{ }^{1} S$, or with the $1 s 2 s{ }^{1} S$ metastable state, so as to simultaneously excite two ${ }^{1} P^{o}$ states, labeled by $2 s 2 p$ and $2 p 3 d$ configurations and undergoing hyperfast "electron correlation beats":

$$
H e\left[\begin{array}{c}
1 s^{2}{ }^{1} S \\
o r, 1 s 2 s^{1} S
\end{array}\right] \begin{aligned}
& \text { femtosecond pulse } h v_{1} \\
& \text { femtosecond pulse } h v_{2}
\end{aligned}\left[\begin{array}{c}
H e(2 s 2 p+1 s \varepsilon p)^{1} P^{o} \\
H e(2 p 3 d+1 s \varepsilon p)^{1} P^{o}
\end{array}\right] .
$$

This system was chosen so as to define "bright", "dark", "gray", and "bath" electron configurations with respect to excitation, all playing roles in the overall physics [14].

I note that, according to the SPS theory of resonance states [21], the labels " $2 s 2 p$ " and " $2 p 3 d$ " correspond to two-electron wavefunctions that include the localized electron correlations to a high degree of accuracy.

One of our proposals, which was supported by quantitative results, was that hyperfast electron rearrangements can be recognized in terms of time-dependent geometries of the electron distributions as the dominant orbital configurations labeling the states correlate [3,14,15] (e.g., see [15], Figure 6). Evidently, a similar picture can exist in molecules, where one may expect that, tracked on attosecond scales, the main features of the electron distribution undergo some degree of oscillation from one nucleus to another.

In summarizing the work of [14], we wrote:

"The determination of the period of population transfer between the two main configurations is one of the crucial results of the present theory, for it suggests that a laser-generated probe in the attosecond range, which will excite (de-excite) the bright or dark configuration state to a higher (lower) state that can be registered, will allow the recording of the motion of two electrons as they occupy different configurations at different times. We note that the geometry of such configurations can be calculated in terms of appropriate mean values and of angular and radial probability densities." ([14], p. L276).

The possibility of time-resolving, on hyperfast scales, the consequences of electron correlations and rearrangements in atoms and molecules is now an area of research that attracts interest both theoretically and experimentally. For example, the significance of the possibility of determining results of orbital rearrangements in molecules in future measurements with attosecond resolution was argued 
by Krausz in a recent review [9]. I quote from Section 6 of [9], which is titled "Future prospects: exploring electronic motions in complex systems":

However, there is another observable of crucial importance: the expectation value of the electrons' spatial coordinates. Dynamic changes in the electrons' atomic-scale distribution ... atomic-scale rearrangements of electron charge distributions occurring on the electronic timescale remain inaccessible in complex systems, such as biological molecules and solid-state nanostructures, to this day. Making them perceivable requires attosecond-duration multi-keV photon (X-ray) or electron pulses, permitting diffraction imaging with picometer spatial and attosecond temporal resolution.

From the point of view of theory, fulfilling this prospect will require quantitatively time-resolving the occupation of orbital configurations in different geometries, analogous to the results for intra-atomic electron rearrangements [15].

\section{Photoionization with Attosecond Resolution. Measurements on Neon and on Helium Agree with Theoretical Predictions}

I now turn to two new phenomena that were originally revealed during the '00s in studies of photoionization of helium and neon. Thus far, their understanding has been possible not only descriptively, but also quantitatively, thanks to achievements at both the experimental and the theoretical levels.

As regards experiment, the development and implementation of novel pump-probe techniques have allowed the measurement of quantities that describe time-dependent electron dynamics with attosecond resolution. As regards theory, the same quantities were understood and predicted accurately, thanks to the development and implementation of formalisms that allow the calculation and use of the $\Psi(t)$ that is appropriate for the solution of the problem. The solution of the METDSE has been obtained either nonperturbatively via the SSEA, or to first order in perturbation theory. In the latter case, we showed that the results of interest can be obtained from an analytic formula that uses $\mathrm{N}$-electron wavefunctions and matrix elements [16].

Only a description of the essentials is given here. The main purpose is to point out that the experimental findings on helium $[7,8]$ and neon $[10,11]$ are in agreement with our theoretical results of 2007 [16] and 2010 [11] (see also the conclusion of [17]). Complete information can be found in the original publications $[7,8,10,11,16]$, including the supporting online material of [11]. The same themes were also briefly reviewed in [3], which is based on an invited talk at the 2015 Sanibel conference.

6.1. Time-Resolved Generation of the Photoionization Cross-Section Profiles of Doubly Excited and Inner-Hole Autoionizing N-Electron States on Hypershort Time Scales

In the mid-'00s, in continuation of the initial work on attosecond electron dynamics $[14,15]$ discussed in Section 5, we completed two projects along the same direction. The corresponding publications of $2007[6,16]$ contain theory and quantitative predictions pertaining to hyperfast electron processes of excitation and decay of autoionizing states, initiated by the interaction of femtosecond EUV pulses with the closed-shell $\mathrm{He} 1 s^{21} S$ state [16] and the open-shell $A l K L 3 s^{2} 3 p^{2} P^{o}$ state [6].

In the aluminum case, photoionization by the pulsed interaction creates the 2s-hole autoionizing states $\left(1 s^{2} 2 s 2 p^{6} 3 s^{2} 3 p\right)^{3} P^{o}$ and ${ }^{1} P^{o}$. Because of the open valence shell, the different electron couplings result in different self-consistent fields and electron correlations for the two states. Hence, the dynamics are channel-dependent. For reasons of economy, I will not discuss this case here. The reader is referred to the original paper [6] and the review [2].

In the helium case, the pulsed interaction causes the two-electron excitation above the ionization threshold,

$$
\text { He } 1 s^{2}{ }^{1} S \rightarrow(2 s 2 p){ }^{1} P^{o}(\mathrm{~A}) .
$$

The label $(2 s 2 p){ }^{1} P^{o}$ represents the zero-order bound configuration of the resonance state inside the 1 sep ${ }^{1} P^{o}$ continuous spectrum at about $60.1 \mathrm{eV}$ above the ground state. The energy-dependent 
stationary description of the resonance is a superposition of the correlated localized component corresponding to $(2 s 2 p){ }^{1} P^{o}$, with the energy-normalized scattering components $1 \operatorname{sep}{ }^{1} P^{o}$.

The helium project extended the theory and calculations of [14] in order to rigorously treat, from first principles, a novel problem of time-dependent many-electron physics, namely tracking the creation of a resonance state (scheme A) as this is recorded by the time-dependent appearance of the cross-section resonance profile inside the continuous spectrum. The much more demanding work on $\mathrm{Al}[6]$ also produced results on this phenomenon.

The work was inspired by the 2005 publication of Wickenhauser et al. [52] who, using a model system that assumes data from (super) Coster-Kronig transitions with lifetimes of $\sim 400$ attoseconds, "investigated the feasibility of observing the buildup of a Fano resonance in the time domain by attosecond streaking techniques" ([52], abstract), and produced results on the phenomenology of such a possibility.

The theory and calculations in [16] were developed and carried out within two frameworks. One involved nonperturbatively solving the METDSE via the SSEA, which is valid for weak as well as strong fields. The other was based on a new time-dependent approach, whereby the METDSE is solved to first order in perturbation theory, while employing the Fano description of resonance states on the real energy axis. In both cases, the calculation is done ab initio, using accurate wavefunctions and matrix elements.

The results were obtained for a $\sin ^{2}$ pulse of duration 450 a.u $(11 f s)$ and central energy of about $60.1 \mathrm{eV}$. (The lifetime of the $\mathrm{He}(2 s 2 p){ }^{1} P^{o}$ resonance is about $17 f \mathrm{f}$.) They were depicted as snapshots of $P(\varepsilon, t) \equiv \mid<$ scattering state $(\varepsilon)\left|\Psi_{S S E A}(t)>\right|^{2}$, where $\varepsilon$ is the energy above the ionization threshold, at various femtosecond intervals. They showed quantitatively how, as the two electrons in the bound orbitals $2 s$ and $2 p$ are allowed to interact with the scattering continuum, the interference of the bound and scattering components evolves until the formation of the resonance asymmetric profile on the energy axis is completed. Upon completion of this profile, the agreement with the experimental values and the earlier theoretical results on the energy axis (energy position, width, and the Fano $q$ parameter for the profile) is excellent [16].

The aforementioned analytic treatment, which is valid for weak fields only, produced a practical analytic formula (corrected for a misprint in [16]) that uses $\mathrm{N}$-electron matrix elements and incorporates the pulse characteristics. It was demonstrated in [16] that when the field is weak, the numerical results from applying this formula are identical to those from the nonperturbative SSEA calculation ([16], Figure 2). The formula can be used for appropriate predictions in many-electron atoms, provided its parameters are known from computation or experiment. For example, its application shows that Auger states of atoms in the middle of the periodic table, whose width is sufficiently large, about $2 \mathrm{eV}$ or more, are suitable candidates for observing the formation of their stationary profiles with attosecond duration.

The predictions for $A l$ have not yet been tested experimentally. However, those for helium were indeed subjected to a novel pump-probe experiment with a time delay of sub-femtosecond precision $[7,8]$. The findings and analysis of $[7,8]$ confirmed the theory of [16], although the experiment did not cover the full duration of the generation of the complete resonance profile of the $(2 s 2 p)^{1} P^{o}$ state on the energy axis, which was calculated to be about 180 femtoseconds (i.e., about 10 lifetimes) [16].

Given the continuing experimental progress on refined measurements on hypershort time scales, we expect that novel experiments such as those of [7,8] (see also [53]) can be developed further, in order to allow the study of even more intriguing time-resolved processes involving multiply excited or inner-hole-excited states inside the continuous spectrum. Such states are normally unstable (autoionizing). However, due to symmetry restrictions, it is also possible for a few of them to belong to the nonrelativistic discrete spectrum in spite of their very high excitation. For example, as a prototypical case we have treated aspects of the time-dependent dynamics of the preparation of the bound triply excited state of a negative ion, $\mathrm{He}^{-} 2 p^{3}{ }^{4} S^{o}$, which is $59.33 \mathrm{eV}$ above the helium ground state, using 
two pulses with time delay [54]. The wavelengths of the two pulses resonantly connecting the states are in the infrared $(10,080 \AA)$ and soft x-ray (323 $\AA$ ) regions. The ladder of excitation is [54],

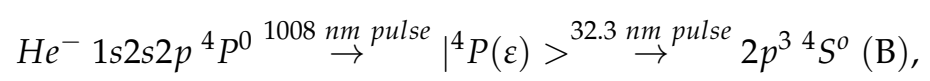

where $1 s 2 s 2 p^{4} P^{0}$ is metastable and $\mid{ }^{4} P(\varepsilon)>$ is a shape resonance labeled by the zero-order $\left(1 s 2 p^{2}\right)^{4} P$.

\subsection{Relative Time-Delay in the Photoemission of Electrons from Different Subshells}

\subsubsection{Introduction}

In 2008, experimentalists at the department of attosecond physics of the Max Planck Institute of Quantum Optics in Garching, Germany, headed by F. Krausz, were in the process of carrying out a spectacular pump-probe experiment of fundamental significance. This involved the implementation of their attosecond streaking technique for the photoionization of neon. Their goal was to measure whether, upon photoemission of the electrons from the $2 s$ and $2 p$ subshells of neon by an attosecond pulse with photon energy centered around $106 \mathrm{eV}$, the electrons are ejected absolutely simultaneously, as the conventional wisdom was at the time, or with a time difference, i.e., with a relative time delay (TD).

In view of that experimental project, V. Yakovlev of the Garching institute and the Athens team started a collaboration with the purpose of developing a many-electron theory that could determine whether such a TD could indeed occur in neon photoionization, and, if so, what its sign and magnitude ought to be. This possibility would be unique in the study of atomic physics, since there are no other collision processes (e.g., other atoms) that could interfere.

At that time we were in the dark, since this new problem of time-dependent many-electron quantum mechanics had never been considered and understood theoretically, let alone solved quantitatively. The only certainty was that if this were a real phenomenon, the corresponding information ought to be contained in the METDSE describing the synchronized pump (EUV attosecond pulse) plus probe (IR laser pulse) experiment, in terms of the Hamiltonian $\boldsymbol{H}_{t o t}(t)=\mathbf{H}_{\mathrm{Ne}}+\boldsymbol{V}_{\text {int }}(t)$. $\mathbf{H}_{N e}$ is the Hamiltonian of the field-free neon and $V_{\text {int }}(t)=V_{I R}(t)+V_{E U V}(t-\Delta t)$, where $\Delta t$ is the controlled time delay between the application of the two pulses in the attosecond streaking experiment [11].

The theoretical treatment and results were published in [11], including its SOM (see also [17] and below), together with the experimental findings of Schultze et al. [11]. Both the experiment and the theory introduced into atomic and molecular physics a new and observable concept in attosecond electron dynamics-namely the relative TD in the photoemission from atoms-and methods in terms of which quantitative results can be obtained.

After the 2010 publication [11], a large number of publications, experimental as well as theoretical, have dealt with aspects of this problem, not only for neon but also for other cases. This body of work was recently reviewed comprehensively by Pazourek, Nagele, and Burgdörfer [55]. Section V of [55], titled "Time-resolved photoionization of many-electron atoms", contains a discussion related to the seminal work on neon. As regards theory, even time-independent treatments have been implemented, reducing the understanding of a phenomenon where the concept of time is inevitably involved (e.g., quantum mechanical time of free $\mathrm{N}$-electron wavepacket formation), to the calculation of photoionization Eisenbud-Wigner-Smith (EWS) time delays in terms of energy derivatives of scattering phases.

\subsubsection{Elements of the Theoretical Model and Results of Schultze et al. on Neon}

The $(2 s, 2 p)$ photoionization of neon by the attosecond pump pulse centered at about $106 \mathrm{eV}$ with a width of about $14 \mathrm{eV}$ involves the dipole transitions $2 s \rightarrow \varepsilon p, 2 p \rightarrow(\varepsilon s, \varepsilon d)$ and ${ }^{1} p^{o}$ final states in the continuum. This picture excludes two-electron excitations. In the presence of the probe 
$V_{I R}(t)$, states with many angular momenta in the continuum are mixed. These states are labeled as $\psi_{2 s}^{\ell, L}(\varepsilon)=\left(1 s^{2} 2 s 2 p^{6}\right) \varepsilon \ell^{1} L$ and $\psi_{2 p}^{\ell^{\prime}, L^{\prime}}(\varepsilon)=\left(1 s^{2} 2 s^{2} 2 p^{5}\right) \varepsilon \ell^{\prime 1} L^{\prime}$.

If we were to use the SSEA with $\boldsymbol{H}_{t o t}(t)$, the corresponding $\Psi_{S S E A}(t)$ would be constructed in the following form:

$$
\Psi_{S S E A}(t)=a_{g_{s}}(t) \Phi_{g s}\left({ }^{1} S\right)+\sum_{\ell, L}^{\ell=\ell_{\max }} \int^{\varepsilon_{\max }} b_{2 s}^{\ell, L}(\varepsilon, t) \psi_{2 s}^{\ell, L}(\varepsilon) d \varepsilon+\sum_{\ell^{\prime}, L^{\prime}}^{\ell^{\prime}=\ell^{\prime} \max } \int^{\varepsilon_{\max }} b_{2 p}^{\ell^{\prime}, L^{\prime}}(\varepsilon, t) \psi_{2 p}^{\ell^{\prime}, L^{\prime}}(\varepsilon) d \varepsilon
$$

According to the SSEA methodology, the upper limits of the above summations, $\ell_{\max }$ and $\ell_{\text {max }}^{\prime}$, as well as the upper limits of the integrals $\varepsilon_{\max }$, are increased until full convergence of the time-dependent complex coefficients is achieved. The bound wavefunctions of the ground state, $\Phi_{g s}\left({ }^{1} S\right)$, and the ionic cores are computed by including the few single and double orbital electron correlations, which contribute according to the arguments and methods of the SPS theory.

The complex coefficients of the SSEA are probability amplitudes with physical meaning, containing quantum mechanical information about transitions to each energy-normalized scattering state to which they correspond. By asserting that photoionization is a half-collision process, it is reasonable to invoke the concept of time-delay developed in scattering theory and associated with the names Eisenbud, Wigner, and Smith (EWS) [55-57]. Accordingly, the time delay for each channel is obtainable from $\hbar \frac{d}{d \varepsilon} \arg \left[b_{2 s}^{1,1}(\varepsilon, t)\right]$ and $\hbar \frac{d}{d \varepsilon} \arg \left[b_{2 p}^{2,1}(\varepsilon, t)\right]$ in the energy region fixed by the EUV excitation energy. For neon, as well as for other systems, a quantitative understanding of the degree of sensitivity of these quantities (energy derivatives of scattering phases) on the wavefunctions and the resulting complex coefficients is a crucial element of theoretical work in this new area of research.

The theoretical treatment in [11] did not apply the SSEA with the Hamiltonian containing both EUV and IR interactions, since this was computationally prohibitive for our computer facilities. Instead, the problem was separated into two parts, which were put together by Yakovlev and collaborators in Garching.

The second part was conceived and carried out in Garching. It involved implementing the one-electron Coulomb-Volkov approximation (CVA) to the estimation of the streaking delay caused by the interaction of the outgoing wavepackets with the IR laser pulse.

The first part had to do with the many-electron solution of the METDSE via the SSEA using only the EUV pump interaction, with the purpose of determining the relative TD for the one-photon processes,

$$
\begin{aligned}
& \text { Ne } 1 s^{2} 2 s^{2} 2 p^{6}{ }^{1} S \\
& \text { atto sec ond pulse } \\
& h v \approx 106 \mathrm{eV}
\end{aligned}\left[\begin{array}{c}
N e^{+} 1 s^{2} 2 s 2 p^{6}{ }^{2} S+\varepsilon p \\
o r, N e^{+} 1 s^{2} 2 s^{2} 2 p^{5} P^{o}+\varepsilon S, \varepsilon d
\end{array}\right] \text { (C). }
$$

Systematic calculations using the parameters of the experimental attosecond pulse determined the effects on the SSEA scattering coefficients and on the corresponding TD, of the state-specific self-consistent fields, of the important state-specific electron correlations (according to the SPS theory $[21,22])$, of interchannel coupling, and of resonance states corresponding to two-electron excitations from the 2 s subshell $[3,11,17]$.

The theory and results of [11] (see below) corroborated the essence of the experimental findings. Specifically, it was demonstrated that upon photoabsorption, assumed to occur instantaneously, the emergence of the wavepackets (of different kinetic energies) representing the electrons initially occupying the neon $2 p$ and $2 s$ HF orbitals takes place with a relative TD. In agreement with the experiment, the $2 p$ electron emerges a few attoseconds after the $2 s$ electron does.

The SSEA result for the spectrally averaged relative TD of the electron wavepackets emitted according to scheme (C), including doubly excited resonances (see below) was 6.38 as. It was impressive that the same number (6.37 as) was obtained independently from the simulation of the streaking measurement in terms of the CVA ([11], page 6 of the SOM). 
On the other hand, the contribution of the IR-laser perturbation on the emitted electrons, deduced according to the CVA [11], was $\mathbf{2 . 3}$ as. (The text in [11] reads: "Thus, for the current experimental parameters, the small deviation between the exact electron's motion and the modeled via the CVA gives rise to a two-attosecond discrepancy in the relative delay. Accepting this small discrepancy, many-electron models were applied to investigate the effects of electron correlation" (p. 1661).

Therefore, as regards the simulation of the streaking measurements of [11], the theoretical prediction for the spectrally averaged relative TD for neon was

$$
[6.4 \text { as }(\mathrm{XUV}-\mathrm{SSEA})+\mathbf{2 . 3} \text { as }(\mathrm{IR}-\mathrm{CVA})]=\mathbf{8 . 6 7} \text { as } \quad \text { (Theory of [13]) }
$$

This pump + probe number, or just the SSEA one, the latter representing what was called in [11] the "Wigner-Smith" TD for the process of photoionization, was in quantitative disagreement with the measurement of [11], which gave $\mathbf{2 1} \pm \mathbf{5}$ as.

In the next subsections, I focus on three themes related to [11]. The first has to do with the possible role of doubly excited resonances, whose existence at the time of the experiment was unknown. The second has to do with the standard question of the degree of agreement between the theoretical and experimental results. The third has to do with information that only theory can provide, namely, how to understand this phenomenon in neon in terms of the interplay between interelectronic interactions (i.e., wavefunctions) and dynamics (i.e., transition amplitudes).

\subsection{The Relative Time Delay in the Neon Problem: The Role of Doubly Excited Resonances}

The quantitative disagreement between theory and experiment persisted in the course of the investigations on the relative TD of neon in Athens and Garching. Both experimentalists and theoreticians were confident that their results were obtained correctly. In this context, the Athens team considered the possibility that double excitations reaching resonances that were unknown at the time might be responsible for at least some of the observed discrepancy.

Given the energetics and the nature of the electronic structure, we concluded that good candidates ought to be states that are created by double excitations from the 2s subshell [17]. Although we established the existence of a series of resonances with a double hole in the $2 s^{2}$ subshell, of which one or two have their positions at the experimentally accessible energy region of the photoelectron, the conclusion as to their influence was negative because of their small width in relation to the spectral averaging over the large width of the attosecond pulse (see the conclusion of [17]).

By inference, in other cases of photoionization, the relative TD might indeed be affected by the presence of a very wide scattering resonance, or of closely lying resonances, at the energy where the photoelectron is emitted. For example, the dependence of time delays on resonance states was examined recently by Sabbar et al. in studies of neon and argon [58], and by Dolmatov et al. in theoretical studies of the photoionization of Mn [59].

\subsubsection{The Relative Time Delay in the Neon Problem: Comparison of Theory with Experiment}

The quantitative discrepancy between the experimental and theoretical results reported in [11] evidently presented a scientific challenge. On page 465 of [3], I commented as follows:

"The enigma stemming from the reported difference between the experimental and the theoretical findings in [11] remains to date, in spite of subsequent theoretical investigations by a number of researchers. It is challenging to both theory and experiment. In this context, it is worth noting that the same theoretical number, [to that of the SSEA], i.e., 6.4 as, was reported recently by Feist et al. [60] who carried out very large R-matrix type calculations, including a series of resonance states near and far from the critical region of $106 \mathrm{eV}$. Their results confirm the original theoretical predictions which were made in [11,17]."

The "enigma" stated above was resolved recently with the publication by Isinger et al. [10] of new measurements with high spectral resolution, which allowed the disentanglement of the direct $2 \mathrm{~s}$ 
ionization from the two-electron process of ionization plus excitation to a $3 p$ state [10]. The qualitative conclusions of Isinger et al. [10] as to the sequence of emission of electrons from the $2 s$ and $2 p$ subshells of neon confirm the original experimental and theoretical predictions of Schultze et al. [11].

However, the new experimental number [10] is about $\mathbf{1 0}$ as, much closer to the theoretical result first predicted via the theory and the SSEA calculation in [11] than to the experimental value [11].

Here, a parenthetical clause is necessary. The authors of [10] present the discrepancy between the theoretical and experimental TD as a "puzzle" that came to the fore after calculations that followed the publication of [11] produced such a result. (The theoretical work in [10] used the many-body perturbation theory.) In fact, this was already known and reported explicitly, with specific numbers, in the seminal publication [11], which demonstrated, conceptually and quantitatively, the presence of the relative TD in neon, both experimentally and theoretically. (See the paragraph from [3] quoted above.) The calculations by other groups that appeared after the publication of [11], (quoted in [10]), simply confirmed its theoretical finding, which was obtained based on the analysis outlined in Section 6.2.2. It is gratifying to see that, once the phenomenon was understood and presented in [11], later large-scale calculations by Feist et al. [60], Moore et al. [39], and others, produced a number that is the same as, or very close to, the theoretical numbers (6.4 as and 8.7 as) reported in [11].

\subsubsection{The Relative Time Delay in the Neon Problem: Self-Consistent Orbitals and Electron Correlation}

I quote from [3], page 464:

"Contrary to our initial anticipation, the results for this system did not show a strong dependence on details of electron correlation beyond the MCHF level, or on interchannel coupling. For example, whereas the final result due to the pump step was 6.4 as, the result without interchannel coupling, i.e., using just the dipole matrix elements connecting the initial state-specific orbitals, calculated at the MCHF level, to their corresponding final state channel, was 4 as (see page 1661 of [11])."

In other words, we found that the cause of the bulk of the relative $(2 s, 2 p)$ TD in neon is already produced at the level of state-specific HF-MCHF wavefunctions for initial and final states, even though the hole-filling electron correlation, $2 p^{2}-2 s \bar{d}$, normally important for transition processes is present only in the case of photoionization from the $2 s$ subshell. $\bar{d}$ is a one-electron function of $d$ symmetry representing the sum of the $d$ orbitals of the discrete and the continuous spectrum calculated in the term-specific N-1-electron HF potential.

Nevertheless, the fact that the theoretical results (6.4 as, or 8.7 as with streaking) differed from the experimental value ( $21 \pm 5$ as) was later assessed in some publications as being caused by omitted electron correlations of some unknown type.

For example, soon after the publication of [11], Sansone [61], commenting on its results, wrote:

“... more refined models including multi-electron correlation, such as the state-specific expansion approach. Even though these models turn out to be in qualitative agreement with the experimental observation (the photoionization from the $2 p$ shell is delayed with respect to that from the $2 s$ shell), they do not offer proper agreement with the experiment as the estimated delay of 5 as is three standard deviations smaller than the measured delay. The discrepancy clearly indicates that our understanding and capability of modelling multi-electron correlation effects is still far from being complete and that subtle effects leading to attosecond time delay cannot yet be satisfactorily described. In this contest, the experimental data represents a benchmark for more sophisticated and advanced models including electron correlations."

In the above quotation, the "blame" for the discrepancy between theory and experiment that was reported in [11] was placed on the ostensible lack of "understanding and capability" of handling electron correlation effects.

Along a similar line of reasoning on the significance of electron correlations in determining the relative TD in neon is the statement made in the commentary by Day [62] on the important advances in physics of the past few years, where this TD is attributed to "subtle electron-electron correlations". 
On the other hand, the authors of the recent publication [10] have stated that according to their calculations, the effect of electron correlation on the neon TD is rather small compared to the result based on the zero-order orbital approximation, thereby confirming our conclusion from the original theoretical work [11].

Of course, as in many other properties or phenomena, only when the important electron correlation effects are accounted for is the theoretical understanding reliable.

In conclusion, our calculations and analysis of the neon TD problem [11] showed that the phenomenon is due mainly to the different state-specific self-consistent fields, and that electron correlation in the bound states and interchannel coupling in the continuous spectrum act as correction factors. This is why, in the time-independent framework of calculating "Wigner time delays", algorithms such as the random phase approximation and limited many-body perturbation theory have produced similar numbers for the closed-shell neon ${ }^{1} S$ state.

However, in general, for $\mathrm{N}$-electron systems whose electronic structures and spectra are quite different than those of neon, the contributions to the energy derivatives of the phases of the complex coefficients of the scattering channel in the photoelectron energy of interest, and therefore to the relative $\mathrm{TD}$, may indeed depend significantly on electron correlations and interchannel couplings, or on the presence of broad resonances.

\section{Conclusions}

Thanks to significant developments of the last two to three decades, both theory and experiment can now provide the same or complementary information and insight into attosecond-resolved electron dynamics associated with hyperfast electron rearrangements upon pulsed excitation of atoms and molecules into the continuum.

As regards experiment, the novel techniques that have been developed and used for the study of such phenomena are discussed in the cited works [7,8,10,11,29].

As regards theory and computation, the formal construction, the credible solution, and the quantitative understanding of the related new types of TDMEPs require that the METDSE is treated and solved from a many-electron point of view, for closed- as well as for open-shell atomic or molecular states, rather than in terms of models or one-electron potentials. To this purpose, a fundamental requirement is for theory to account for the details of the electronic structures and the scattering continua of the states participating in the dynamics.

The SSEA, whose main features were presented in Section 3, satisfies the above requirements [1-6,14-16,21,22]. Indeed, because of its structure, its implementation for each problem of interest can be easily understood, regardless of the wavelength(s) of the applied pulse(s), which can range from the IR to the x-ray region.

In Sections 4-6, I discussed various types of TDMEPs whose solutions, with attosecond resolution, have provided quantitative information and good insight into hyperfast electron dynamics and their relation to state-specific self-consistent fields, electron correlations, interchannel couplings, and resonances. The results were obtained in terms of many-electron wave functions $\Psi(t)$, calculated by solving the METDSE nonperturbatively via the SSEA. In addition, when the pulse is chosen to be short and relatively weak, it is possible to formulate and calculate perturbatively the time-resolved preparation and evolution of states in the continuum in terms of practical analytic formulas containing information about the characteristics of the pulses and the time delay between two pulses $[16,54,63]$.

The cases that were brought to attention as examples were:

(1) The ab initio determination of the details of the time-resolved decay of autoionizing states via one-electron tunneling or two-electron rearrangements, thereby identifying quantitatively the regimes of nonexponential decay (NED) for states very close to threshold. I point out that the predictions for NED were made not only for the case of very long times [12,13] but also for the case of very short times after the preparation of the N-electron decaying state [64]. The degree to which the 
preparation step affects the NED for each particular N-electron isolated decaying state might be a challenging question for attosecond physics.

(2) The time-resolved coherent preparation and decay of strongly correlated doubly excited and inner-hole excited autoionizing states. (Prototypical cases of electron dynamics in helium and aluminum.)

(3) The time-resolved identification of electron correlation beats and correlation-induced geometrical rearrangements of electrons inside the atom, which occur on the attosecond scale. For molecules, such rearrangements may take also place interatomically.

(4) The time-resolved generation of the energy profiles of doubly excited (helium) and inner-hole excited (aluminum) autoionizing states during photoionization by hypershort radiation pulses. The published figures explicitly and quantitatively depicted for the first time the time-dependent effects on these profiles of the interference between the localized and scattering components of these states.

(5) The relative time delay in the photoemission of two electrons from different subshells of an atom (case of the $(2 s, 2 p)$ photoionization of neon).

The information that has been obtained from the results of our treatment of these prototypical TDMEPs agrees with what has been produced via novel experiments capable of attosecond resolution $[7,8,10,11]$.

In accordance with the original proposal and results [14,15], in all the above examples the hyperfast imaging of electron dynamics reveals the significance of both the state-specific self-consistent orbitals and their dominant electron correlations.

Conflicts of Interest: The author declares no conflict of interest.

\section{References}

1. Mercouris, T.; Komninos, Y.; Dionissopoulou, S.; Nicolaides, C.A. Computation of strong-field multiphoton processes in polyelectronic atoms. State-specific method and application to $\mathrm{H}$ and $\mathrm{Li}^{-}$. Phys. Rev. A 1994, 50, 4109. [CrossRef] [PubMed]

2. Mercouris, T.; Komninos, Y.; Nicolaides, C.A. The state-specific expansion approach to the solution of the polyelectronic time-dependent Schrödinger equation for atoms and molecules in unstable states. Adv. Quantum Chem. 2010, 60, 333-405.

3. Nicolaides, C.A. Quantum chemistry on the time axis: Electron correlations and rearrangements on femtosecond and attosecond scales. Mol. Phys. 2016, 114, 453-468.

4. Nicolaides, C.A.; Dionissopoulou, S.; Mercouris, T. The significance of electron correlation and of state symmetries in the interaction of strong laser pulses of $5 \mathrm{eV}$ with He. J. Phys. B At. Mol. Phys. 1998, 31 , L1. [CrossRef]

5. Mercouris, T.; Komninos, Y.; Nicolaides, C.A. EUV two-photon-ionization cross sections of helium from the solution of the time-dependent Schrödinger equation, and comparison with measurements using free-electron lasers. Phys. Rev. A 2016, 94, 063406. [CrossRef]

6. Mercouris, T.; Komninos, Y.; Nicolaides, C.A. Time-resolved hyperfast processes of strongly correlated electrons during the coherent excitation and decay of multiply excited and inner-hole states. Phys. Rev. A 2007, 76, 033417. [CrossRef]

7. Kaldun, A.; Blättermann, A.; Stooß, V.; Donsa, S.; Wei, H.; Pazourek, R.; Nagele, S.; Ott, C.; Lin, C.D.; Burgdörfer, J.; et al. Observing the ultrafast buildup of a Fano resonance in the time domain. Science 2016, 354, 738-741. [CrossRef] [PubMed]

8. Blättermann, A.; Kaldun, A.; Stooß, V.; Donsa, S.; Wei, H.; Pazourek, R.; Nagele, S.; Ott, C.; Lin, C.D.; Burgdörfer, J.; et al. Watching the emergence of a Fano resonance in doubly excited helium. J. Phys. Conf. Ser. 2017, 875, 012010. [CrossRef]

9. Krausz, F. From quantum transitions to electronic motions. Appl. Phys. B 2017, 123, 7. [CrossRef] 
10. Isinger, M.; Squibb, R.J.; Busto, D.; Zhong, S.; Harth, A.; Kroon, D.; Nandi, S.; Arnold, C.L.; Miranda, M.; Dahlström, J.M.; et al. Photoionization in the time and frequency domain. Science 2017, 358, 893-896. [CrossRef] [PubMed]

11. Schultze, M.; Fieß, M.; Karpowicz, N.; Gagnon, J.; Korbman, M.; Hofstetter, M.; Neppl, S.; Cavalieri, A.L.; Komninos, Y.; Mercouris, T.; et al. Delay in photoemission. Science 2010, 328, 1658-1662. [CrossRef] [PubMed]

12. Nicolaides, C.A.; Mercouris, T. On the violation of the exponential decay law in atomics physics: Ab initio calculation of the time-dependence of the $\mathrm{He}^{-} 1 s 2 p^{2}{ }^{4} \mathrm{P}$ nonstationary state. J. Phys. B At. Mol. Opt. Phys. 1996, 29, 1151. [CrossRef]

13. Mercouris, T.; Nicolaides, C.A. Time dependence and properties of nonstationary states in the continuous spectrum of atoms. J. Phys. B At. Mol. Phys. 1997, 30, 811. [CrossRef]

14. Nicolaides, C.A.; Mercouris, T.; Komninos, Y. Attosecond dynamics of electron correlation in doubly excited atomic states. J. Phys. B At. Mol. Opt. Phys. 2002, 35, L271. [CrossRef]

15. Mercouris, T.; Komninos, Y.; Nicolaides, C.A. Theory and computation of the attosecond dynamics of pairs of electrons excited by high-frequency short light pulses. Phys. Rev. A 2004, 69, 032502. [CrossRef]

16. Mercouris, T.; Komninos, Y.; Nicolaides, C.A. Time-dependent formation of the profile of the He $2 s 2 p{ }^{1} P^{o}$ state excited by a short laser pulse. Phys. Rev. A 2007, 75, 013407, Erratum in 2013, 87, 069905(E). [CrossRef]

17. Komninos, Y.; Mercouris, T.; Nicolaides, C.A. Regular series of doubly excited states inside two-electron continua: Application to $2 s^{2}$-hole states in Neon, above the thresholds $2 N e^{2+} 1 s^{2} 2 s^{2} 2 p^{4}$ and $1 s^{2} 2 s 2 p^{5}$ thresholds. Phys. Rev. A 2011, 83, 022501. [CrossRef]

18. Kulander, K.C. Time-dependent Hartree-Fock theory of multiphoton ionization. Phys. Rev. A 1987, $36,2726$. [CrossRef]

19. Kulander, K.C. Time-dependent theory of multiphoton ionization of Xenon. Phys. Rev. A 1988, $38,778$. [CrossRef]

20. Parker, J.S.; Moore, L.R.; Smyth, E.S.; Taylor, K.T. One- and two-electron numerical models of multiphoton ionization of helium. J. Phys. B At. Mol. Opt. Phys. 2000, 33, 1057. [CrossRef]

21. Nicolaides, C.A. Resonances in the continuum, field-induced nonstationary states, and the state- and property-specific treatment of the many-electron problem. Adv. Quantum Chem. 2017, 74, 149-181.

22. Nicolaides, C.A. State- and property-specific quantum chemistry. Adv. Quantum Chem. 2011, 62, 35-103.

23. Loudon, R. The Quantum Theory of Light, 2nd ed.; Clarendon: Oxford, UK, 1983.

24. Komninos, Y.; Mercouris, T.; Nicolaides, C.A. Theory and computation of electromagnetic transition matrix elements in the continuous spectrum of atoms. Eur. Phys. J. D 2017, 71, 8. [CrossRef]

25. Nicolaides, C.A.; Mercouris, T.; Petsalakis, I.D. Above and below threshold multiphoton dissociation of volcanic ground states. Application to $\mathrm{BeH}^{++}$. Chem. Phys. Lett. 1993, 212, 685-690.

26. Dimitriou, K.I.; Constantoudis, V.; Mercouris, T.; Komninos, Y.; Nicolaides, C.A. Quantum and classical dynamics of a diatomic molecule in laser fields with frequency in the region producing maximum dissociation. Phys. Rev. A 2007, 76, 033406. [CrossRef]

27. Boguslavskiy, A.E.; Mikosch, J.; Gijsbertsen, A.; Spanner, M.; Patchkovskii, S.; Gador, N.; Vrakking, M.J.J.; Stolow, A. The multielectron ionization dynamics underlying attosecond strong-field spectroscopies. Science 2012, 335, 1336-1340. [CrossRef] [PubMed]

28. Miyagi, H.; Morishita, T.; Watanabe, S. Electron scattering and photoionization of one-electron diatomic molecules. Phys. Rev. A 2012, 85, 022708. [CrossRef]

29. Krausz, F.; Ivanov, I. Attosecond physics. Rev. Mod. Phys. 2009, 81, 163. [CrossRef]

30. Wigner, E. On the interaction of electrons in metals. Phys. Rev. 1934, 46, 1002. [CrossRef]

31. Taylor, G.R.; Parr, R.G. Superposition of configurations: The helium atom. Proc. Natl. Acad. Sci. USA 1952, 38, 154-160. [CrossRef]

32. Löwdin, P.-O. Correlation problem in many-electron quantum mechanics. Review of different approaches and discussion of some current ideas. Adv. Chem. Phys. 1959, 2, 207-322.

33. Komninos, Y.; Nicolaides, C.A. Effects of configuration interaction on photoabsorption spectra in the continuum. Phys. Rev. A 2004, 70, 042507. [CrossRef]

34. Komninos, Y.; Nicolaides, C.A. Quantum defect theory for Coulomb and other potentials in the framework of configuration interaction, and implementation to the calculation of ${ }^{2} D$ and ${ }^{2} F^{o}$ perturbed spectra of Al. J. Phys. B At. Mol. Opt. Phys. 2004, 37, 1817. [CrossRef] 
35. Ishikawa, K.L.; Sato, T. A review on Ab initio approaches for multielectron dynamics. IEEE Sel. Top. Quantum Electron. 2015, 21, 8700916. [CrossRef]

36. Burke, P.G.; Burke, V.M. Time-dependent R-matrix theory of multiphoton processes. J. Phys. B At. Mol. Opt. Phys. 1997, 30, L383-L391. [CrossRef]

37. Lysaght, M.A.; van der Hart, H.W.; Burke, P.G. Time-dependent R-matrix theory for ultrafast atomic processes. Phys. Rev. A 2009, 79, 053411. [CrossRef]

38. Moore, L.R.; Lysaght, M.A.; Nikolopoulos, L.A.A.; Parker, J.S.; van der Hart, H.W.; Taylor, K.T. The RMT method for many-electron atomic systems in intense short-pulse laser light. J. Mod. Opt. 2011, 58, 1132-1140. [CrossRef]

39. Moore, L.R.; Lysaght, M.A.; Parker, J.S.; van der Hart, H.W.; Taylor, K.T. Time delay between photoemission from the $2 p$ and $2 s$ subshells of neon. Phys. Rev. A 2011, 84, 061404(R). [CrossRef]

40. Kato, T.; Kono, H. Time-dependent multiconfiguration theory for electronic dynamics of molecules in an intense laser field. Chem. Phys. Lett. 2004, 392, 533-540. [CrossRef]

41. Caillat, J.; Zanghellini, J.; Kitzler, M.; Koch, O.; Kreuzer, W.; Scrinzi, A. Correlated multielectron systems in strong laser fields: A multiconfiguration time-dependent Hartree-Fock approach. Phys. Rev. A 2005, 71, 012712. [CrossRef]

42. Nest, M.; Remacle, F.; Levine, R.D. Pump and probe ultrafast electron dynamics in LiH: A computational study. New J. Phys. 2008, 10, 025019. [CrossRef]

43. Krause, P.; Klamroth, T.; Saalfrank, P. Time-dependent configuration-interaction calculations of laser-pulse driven many-electron dynamics: Controlled dipole switching in lithium cyanide. J. Chem. Phys. 2005, 123, 074105. [CrossRef] [PubMed]

44. Greenmann, L.; Ho, P.J.; Pabst, S.; Kamarchik, E.; Mazzioti, D.A.; Santra, R. Implementation of the time-dependent configuration-interaction singles method for atomic strong-field processes. Phys. Rev. A 2010, 82, 023406. [CrossRef]

45. Ruberti, M.; Decleva, P.; Averbukh, V. Multi-channel dynamics in high harmonic generation of aligned $\mathrm{CO}_{2}$ : Ab initio analysis with time-dependent B-spline algebraic diagrammatic construction. Phys. Chem. Chem. Phys. 2018. [CrossRef] [PubMed]

46. Pindzola, M.S.; Robicheaux, F. Two-photon double ionization of He and $\mathrm{H}^{-}$. J. Phys. B At. Mol. Opt. Phys. 1998, 31, L823-L831. [CrossRef]

47. Majety, V.P.; Zielinski, A.; Scrinzi, A. Photoionization of few electron systems: A hybrid coupled channels approach. New J. Phys. 2015, 17, 063002. [CrossRef]

48. Nicolaides, C.A.; Beck, D.R. Possibility of observing nonexponential decays in autoionizing states. Phys. Rev. Lett. 1977, 38, 683. [CrossRef]

49. Nicolaides, C.A. Comment on 'Is radioactive decay really exponential?' by Aston P.J. Eur. Phys. Lett. 2013, 101, 42001. [CrossRef]

50. Hentschel, M.; Kienberger, R.; Spielmann, C.; Reider, G.A.; Milocevic, N.; Brabec, T.; Corcum, P.; Heinzmann, U.; Drescher, M.; Krausz, F. Attosecond metrology. Nature 2001, 414, 509-513. [CrossRef] [PubMed]

51. Paul, P.M.; Toma, E.S.; Breger, P.; Mullot, G.; Augi, F.; Balcou, Ph.; Muller, H.G.; Agostini, P. Observation of a train of attosecond pulses from high harmonic generation. Science 2001, 292, 1689-1692. [CrossRef] [PubMed]

52. Wickenhauser, M.; Burgdörfer, J.; Krausz, F.; Drescher, M. Time resolved Fano resonances. Phys. Rev. Lett. 2005, 94, 023002. [CrossRef] [PubMed]

53. Blättermann, A.; Ott, C.; Kaldun, A.; Ding, T.; Pfeifer, T. Two-dimensional spectral interpretation of time-dependent absorption near laser-coupled resonances. J. Phys. B At. Mol. Opt. Phys. 2014, 47, 124008. [CrossRef]

54. Komninos, Y.; Mercouris, Th.; Nicolaides, C.A. Ab initio calculation of time-dependent control dynamics in polyelectronic systems involving bound and resonance states: Application to a quartet spectrum of $\mathrm{He}^{-}$. Phys. Rev. A 2008, 77, 013412. [CrossRef]

55. Pazourek, R.; Nagele, S.; Burgdörfer, J. Attosecond chronoscopy of photoemission. Rev. Mod. Phys. 2015, 87, 765. [CrossRef]

56. Froissart, M.; Goldberger, M.I.; Watson, K.M. Spatial separation of events in S-matrix theory. Phys. Rev. 1963, 131, 2820. [CrossRef] 
57. De Carvalho, C.A.A.; Nussenzveig, H.M. Time delay. Phys. Rep. 2002, 364, 83-174. [CrossRef]

58. Sabbar, M.; Heuser, S.; Boge, R.; Lucchini, M.; Carette, T.; Lindroth, E.; Gallmann, L.; Cirelli, C.; Keller, U. Resonance effects in photoemission time delays. Phys. Rev. Lett. 2015, 115, 133001. [CrossRef] [PubMed]

59. Dolmatov, V.K.; Kheifets, A.S.; Deshmukh, P.C.; Manson, S.T. Attosecond time delay in the photoionization of $\mathrm{Mn}$ in the region of the $3 p \rightarrow 3 d$ giant resonance. Phys. Rev. A 2015, 91, 053415. [CrossRef]

60. Feist, J.; Zatsarinny, O.; Nagele, S.; Pazourek, R.; Burgdörfer, J.; Guan, X.; Bartchat, K.; Schneider, B. Time delays for attosecond streaking in photoionization of neon. Phys. Rev. A 2014, 89, 033417. [CrossRef]

61. Sansone, G. Determining the time zero for electron dynamics. ChemPhysChem 2010, 11, 3581-3583. [CrossRef] [PubMed]

62. Day, C. A reporter's look at the progress of science. Phys. Today 2013, 66, 35. [CrossRef]

63. Nicolaides, C.A.; Mercouris, Th.; Komninos, Y. The time-dependent formation of the profile of resonance atomic states and its dependence on the duration of ultra-short pulses from free-electron lasers. Phys. Rev. A 2009, 80, 055402. [CrossRef]

64. Mercouris, Th.; Nicolaides, C.A. Stationarity coefficients and short-time deviations from exponential decay in atomic resonance states. Phys. Rev. A 2002, 65, 012112. [CrossRef]

(C) 2018 by the author. Licensee MDPI, Basel, Switzerland. This article is an open access article distributed under the terms and conditions of the Creative Commons Attribution (CC BY) license (http:/ / creativecommons.org/licenses/by/4.0/). 